\title{
Intercultural doctor-patient communication in daily outpatient care: relevant communication skills
}

\author{
Emma Paternotte Fedde Scheele $^{1,2} \cdot$ Conny M. Seeleman $^{3} \cdot$ Lindsay Bank $^{1}$ - Albert J. J. A. Scherpbier ${ }^{4}$ \\ Sandra van Dulmen ${ }^{5,6,7}$
}

Published online: 16 September 2016

(C) The Author(s) 2016. This article is available at SpringerLink with Open Access.

\begin{abstract}
Introduction Intercultural communication (ICC) between doctors and patients is often associated with misunderstandings and dissatisfaction. To develop ICC-specific medical education, it is important to find out which ICC skills medical specialists currently apply in daily clinical consultations. Methods Doctor-patient consultations of Dutch doctors with non-Dutch patients were videotaped in a multi-ethnic hospital in the Netherlands. The consultations were analyzed using the validated MAAS-Global assessment list in combination with factors influencing ICC, as described in the literature.

Results In total, 39 videotaped consultations were analyzed. The doctors proved to be capable of practising many communication skills, such as listening and empathic communication behaviour. Other skills were not practised, such as
\end{abstract}

Emma Paternotte

emmapaternotte@gmail.com

1 Department of Healthcare Education, OLVG Hospital, Amsterdam, The Netherlands

2 Medical School of Sciences, Vu University Medical Centre, Amsterdam, The Netherlands

3 Department of Social Medicine, University Medical Centre, Utrecht, The Netherlands

4 Institute for Medical Education, Faculty of Health, Medicine and Life Sciences, Maastricht University, Maastricht, The Netherlands

5 NIVEL (Netherlands Institute for health services research), Utrecht, The Netherlands

6 Department of Primary and Community Care, Radboud University Medical Centre, NIjmegen, The Netherlands

7 Faculty of Health Sciences, University College of Southeast Norway, Drammen, Norway being culturally aware and checking the patient's language ability.

Conclusion We showed that doctors did practice some but not all the relevant ICC skills and that the ICC style of the doctors was mainly biomedically centred. Furthermore, we discussed the possible overlap between intercultural and patient-centred communication. Implications for practice could be to implement the relevant ICC skills in the existing communication training or develop a communication training with a patient-centred approach including ICC skills.

Keywords Doctor-patient communication - Intercultural communication - Communication skills - Clinical practice Reflective practice $\cdot$ Communication behaviour $\cdot$ Medical education

\section{What this paper adds}

- Intercultural communication is challenging for doctors.

- It is however sparsely trained.

- Many intercultural communication skills are known.

- Unknown is, however, what intercultural communication skills medical specialists apply and what should be trained more.

- Implications of the study are the possible overlap between patient-centred communication and intercultural communication.

- This could facilitate development and implementation of intercultural communication skills in medical education. 


\section{Introduction}

Effective, patient-centred communication between doctors and patients is essential for delivering high-quality patient care [1]. Competent communication by doctors improves health outcomes, enhances patient satisfaction, and contributes to doctors' job satisfaction [2]. In the context of a multicultural society, however, effective communication could be hindered by cultural differences between the doctor and the patient [3]. Intercultural communication (ICC), which in this article is defined as communication between a doctor of the dominant ethnic origin and an ethnic minority patient, potentially causes misunderstanding and reduces interpersonal interactions, which may lead to a lower quality of care [4]. Napier et al. stated that 'the systematic neglect of culture in health and healthcare is the single biggest barrier to the advancement of the highest standard of health worldwide' [5].

The theoretical fundaments of ICC between doctors and patients have gained attention in the last few years [6-8]. In a recent review, a conceptual framework of influencing factors in ICC is presented. This framework is constructed based on 145 included articles with a variety of evidence about ICC between the doctor and the patient. The factors influencing ICC, such as the role of the family in a conversation, the doctor's awareness of the effects of differences in ethnic background, or the patient's expectations of a conversation with the doctor, were translated into communication skills. These skills are of great importance in daily clinical practice and hence should be implemented in medical education [6].

The importance of the use of certain communication skills depends on the relevance of that skill in the specific context $[9,10]$. In general, however, professional communication requires adaptation to the specific characteristics of the patient and the situation. Therefore, different contexts, such as differences in ethnic origin between the doctor and the patient, should be explicitly addressed $[3,6,7,11,12]$.

While the theoretical knowledge on ICC skills and the necessity of using these skills have been established [6, $8,13]$, several researchers argue that the scientific field of ICC between doctors and patients in real practice is still too small to develop focused training in ICC $[7,14]$ and that just giving feedback does not cover the full picture of skilled medical communication [15]. It is, for example, unknown which ICC skills are already used by doctors and how they are used. To develop knowledge about the ICC skills used, and therefore also the skills that they do not practice properly, there is a need to further explore which of these communication skills are applicable in the clinical setting, and which of them are already being practised [3, 13].
In this paper, we identify which ICC skills medical specialists use in real practice during those moments in the medical visit in which such skills are judged to be relevant. We addressed the following research question: Which ICCinfluencing factors described in literature are recognizable in doctors' communication skills in real practice?

\section{Methods}

\section{Study design}

In this observational study, doctor-patient consultations with ethnic minority patients and doctors of the Dutch ethnicity were videotaped and analyzed. The analysis focused on the doctor's way of communicating and concentrated specifically on whether the ICC skills identified in a recent realist review were applied in daily practice [6].

\section{Setting and participants}

Between September and December 2014, we videotaped conversations of gynaecologists, internists, urologists and orthopaedic surgeons in the outpatient clinics of the Sint Lucas Andreas Hospital in Amsterdam, the Netherlands. This district teaching hospital serves an urban multi-ethnic area. All Dutch medical specialists of the above-mentioned specialties of the Sint Lucas Andreas Hospital were asked to participate. Medical specialists were chosen since they are role models for residents and more experienced in communication with patients. The patients with a non-Dutch origin were included if they had an appointment for a new episode and the patient had not been seen by this doctor for a year or more. These patients could be of any origin and were not a priori selected. They were all referred by a general practitioner. The patients were asked about their place of birth and that of their parents before they were asked to participate. Informed consent of both the doctor and the patient was requested by the first author who then, if informed consent was obtained, installed the camera and left the room. Exclusion criteria were the presence of an interpreter, a patient with Dutch ethnicity, a doctor of nonDutch origin, a follow-up consultation or a consultation that was partly done by somebody else, for example a medical student.

\section{MAAS-Global InterCultural Communication}

Since a validated observation list for ICC did not exist, we combined the MAAS-Global, a validated instrument for assessing patient-centred communication [2, 16, 17], with 
the factors influencing ICC as found in a recent review [6]. Validation of any measure is a permanent process [18]. The strong base of the items scored in theory supports the content validity of the measure. Since the MAAS-Global was developed in the Netherlands to assess communication skills of general practitioner residents in real practice, we considered it a suitable instrument to use for the observation of communication skills of doctors in the hospital [19]. The MAAS-Global has been used in research settings as well [20]. Combining the two protocols, i.e. the factors influencing ICC and the MAAS-Global, was possible because there is an overlap between the categories of the MAAS-Global and those used to classify the influencing factors in the review. The combination of the MAASGlobal and the ICC-influencing factors provided a framework for coding ICC skills, which could then be observed. The resulting observational scale, the MAAS-Global ICC (Appendix), includes 52 communication skills to be analyzed on a dichotomous scale as 'present or absent' and a 4-point Likert scale to indicate the relevance of each skill for the consultation under observation. This observation and analysing is done per section of the communication, e.g. opening or exploration of reason for encounter (Appendix). In the results section, we report on the relevant skills which were present or absent. Because the MAASGlobal ICC is an extensive list, containing many items, the result section includes the communication skills of the MAAS-Global ICC that were found to be relevant as absent or present in at least $40 \%$ of the consultations.

\section{Measures and analysis}

The adapted MAAS-Global ICC was tested on face validity within the project team, which consisted of specialists from different fields of expertise (medical, cultural competence, communication in healthcare, medical education). The first author (EP) observed and analyzed all the included videotaped consultations. The videotaped consultations were also independently observed and analyzed by one of four second observers (CS, LB, LR, TA), who all watched 9-10 videotaped consultations each. After the first independently observed consultation, the intraclass correlation coefficient (Cohen's kappa) was calculated and discussed between EP and each second observer. Thereafter, EP and the second observer independently scored three consultations, and once again the intraclass correlation coefficient was calculated. If the Cohen's kappa was below 0.6, the videotaped consultation and scoring were discussed to check if the observers could reach a higher level of agreement. Before discussion, the mean Cohen's kappa between the observers ranged from 0.47 to 0.59 . After a discussion between the observers, the mean Cohen's kappa ranged from 0.67 to 0.82 . Scoring of the videotaped consultations was analyzed with SPSS 21 .

After each consultation, doctors were asked if they were satisfied about the consultation. Also, doctors had to write down if they had enough time for the consultation.

\section{Results}

In total, 18 doctors were asked to participate and 17 doctors agreed to participate. One doctor refused because he found it difficult to ask his oncology patients. Of these 17 doctors, 69 consecutive patients of non-Dutch origin were asked to participate. Of these patients, 41 gave informed consent. The other 28 patients refused to participate, mostly because of privacy reasons. Two of the 41 videotaped consultations were excluded, one because the doctor was of non-Dutch origin and the other one because the videotape lacked sound.

Table 1 shows the characteristics of the 39 included videotaped consultations. Furthermore, Table 2 presents the relevant communications skills demonstrated by the doctors. Table 3 lists the communication skills that were not used by the doctors but that the observers considered to be relevant in the specific context of an intercultural conversation. After the consultation, all doctors noted that they

Table 1 Characteristics of the videotaped consultations

\begin{tabular}{|c|c|c|c|c|c|c|}
\hline & $\begin{array}{l}\text { Number of consul- } \\
\text { tations }(n=39)\end{array}$ & $\begin{array}{l}\text { Ethnicity } \\
\text { (non-Western }{ }^{\text {a/ }} \\
\text { Western }^{\text {b }) ~} n=39\end{array}$ & $\begin{array}{l}\text { Gender } \\
(\mathrm{M} / \mathrm{F})\end{array}$ & $\begin{array}{l}\text { Mean } \\
\text { age (y) }\end{array}$ & $\begin{array}{l}\text { Mean years of prac- } \\
\text { tice as medical spe- } \\
\text { cialist (y) }\end{array}$ & $\begin{array}{l}\text { Mean length } \\
\text { videos } \\
(\mathrm{min})\end{array}$ \\
\hline Patients included (\%) & - & $32 / 7(85 / 15)$ & $\begin{array}{l}21 / 18 \\
(54 / 46)\end{array}$ & 46.3 & - & - \\
\hline \multicolumn{7}{|l|}{ Specialty of the doctor ${ }^{c}$} \\
\hline Gynaecology \& Obstetrics & 7 & - & $2 / 3$ & 46.0 & 12.4 & 17.4 \\
\hline Internal medicine & 15 & - & $5 / 1$ & 44.3 & 16.0 & 14.6 \\
\hline Urology & 5 & - & $3 / 0$ & 57.7 & 21.0 & 7.8 \\
\hline Orthopaedics & 12 & - & $4 / 0$ & 52.5 & 15.8 & 13.0 \\
\hline
\end{tabular}

${ }^{a}$ Afghanistan, Turkey, Morocco, Surinam, Nicaragua, Nepal, Nigeria, Cuba, Pakistan, China

boland, Great Britain, Germany, Belgium, Australia, Hungary

${ }^{\mathrm{c}}$ Doctors were all of Dutch origin 
Table 2 An overview of skills, present in at least $40 \%$ of the consultations, that the doctors used: present communication skills

Present communication skills ${ }^{\mathrm{a}}$

The doctor ...

\section{Listens}

Demonstrates reliability (being friendly and having an open attitude)

Makes appointments: who, what, when

Takes the time

Has an unprejudiced attitude

Shows empathic behaviour

Applies an adequate time schedule

Gives concrete explanations

Shows respect for the patient

Uses concrete language

Explains referral to other healthcare workers

Listens actively

Shows concern, is inviting and sincere, commiserates by means of eye contact and non-verbal behaviour, shows compassion for the patient

Commiserates with verbal reactions

Has an open attitude (shows possibilities verbal/non-verbal to give the patient space for their story)

Responds to non-verbal behaviour and keywords

Gives information in small amounts

Tries to empathize with the patient's emotions

Explains cause and relation of the complaint within the context of the expectations of the patient

Reflects on the feelings of the patient

Uses different ways to give explanations

Announces stages of the conversation

Treats the patient with care and respect during physical examination

Checks if the patient and/or relatives understand the explanation

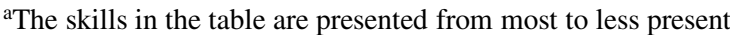

were satisfied with the consultation and that they thought they had enough time for the consultation.

\section{Observed communication skills}

Doctors showed a variety of ICC skills that facilitated the communication. For example, in most consultations doctors adequately employed concrete language, listening and empathic behaviour toward the patients, such as reflecting the patient's feelings and demonstrating concern. Also, doctors gave concrete explanations, for example using drawings to explain an X-ray. Most conversations were not hurried, and most doctors had an adequate time schedule. All these present skills were considered relevant by the observers because, in this way respect, reliability and an unprejudiced attitude were shown.

In many consultations the doctors used a biomedical style of communication, in which they focused on their own agenda with biomedically structured questions and fewer possibilities for the patient to give input.
Table 3 An overview of skills, absent in at least $40 \%$ of the consultations, that the doctors did not use but that were relevant within the context of these consultations: absent communication skills

Absent communication skills ${ }^{\mathrm{a}}$

The doctor did not ...

Check expectations regarding the consultation/healthcare

Ask about the patient's feelings

Ask about the relatives' emotions

Show awareness of his own cultural and professional context

Check foreknowledge of the patient about diagnosis or expected policy

Summarize the patient's story

Explore the reason for the consultation, wishes and expectations

Explore reaction of information transfer to the patient's context

Demonstrate being alert to possible cultural aspects when asking for the reason for the consultation

Show awareness of cultural differences

Show to have learned from previous consultations with ethnic minority patients

Ask if the patient understood the information

Check if the patient and/or family understood the explanation

Adapt cultural differences in diagnosis and policy

Observe cultural differences

Check the language ability of the patient

React adequately to possible cultural differences

${ }^{a}$ The skills in the table are presented from most to less absent

\section{Absent communication skills}

ICC language skills include checking the patient's language ability, which was absent in 17 consultations where it would have been relevant to do. In 37 consultations the main language spoken was Dutch. In two it was English. Absent ICC skills, such as awareness of cultural differences, e. g. the doctor says something about treatment habits in the Netherlands or asks the patient about his/her cultural habits for the specific disease, and adaptation of the diagnosis and treatment policy to the context of the patient, e.g. the doctor asks if the prescription use of the medication is possible and satisfactory for the patient, were considered relevant because these skills facilitate mutual understanding and respect.

These ICC skills were sometimes difficult to score, because they were elusive and not explicit. For example, the doctor did not always directly address a patient's cultural background, but tried to get insight into the patient's perspective by figuring out what the patient thought to be the cause of the complaint (e.g. pain). Also, many doctors did not check the foreknowledge of the patient about the diagnosis and treatment policy. The relevance of attention to cultural differences was emphasized in the doctor's explicit communication. For example, doctors did not take the patient's context into account when proposing a policy, 
such as medication intake or dietary advice, and they had difficulties shifting from their biomedical communication style to the context and expectations of the patient. When the conversation was mainly biomedical, it was difficult to determine if the doctors were aware of their own cultural and professional context. In a few conversations the doctors mentioned their own cultural origin, for example by explaining how a treatment is carried out in the Netherlands. This, however, did not linearly cause doctors to pay attention to cultural differences. Summaries were not often used in the conversation, although this could have structured the conversation and it could have helped both the doctor and the patient to check if specific information was understood correctly.

Other skills that were absent but relevant lay in the field of expectation management, showing interest in the patient's family and checking if the patient understood the information given by the doctor, which was relevant as it might have helped to clarify possible misunderstandings. An example of expectation management is exploring the patient's view on the reason for the consultation or the patient's expectation of the consultation. However, if doctors used questions aimed at clarifying the patient's expectations, which was not done in $62 \%$ but used in $38 \%$ of the consultations, this proved to facilitate ICC and direct the communication into a more patient-centred approach, depending on the way they were phrased. For example, after listening to a complex account of the patient's complaints, one doctor asked: 'What do you expect from me? Would you like me to reduce the pain, or is it something else?'

\section{Discussion}

In this observational study, we focussed on relevant ICC skills of medical specialists in real practice. The medical specialists in this study proved to be capable of practising many communication skills, such as listening, showing empathic communication behaviour and being open and respectful to the patient. Other skills were not practised although they were relevant in the intercultural context, such as being culturally aware, checking the patient's language ability, checking if the patient understood and exploring the reason for the consultation. The communication style of the doctors was often a biomedical style.

The use of a biomedical style in these intercultural conversations is surprising, since ICC requires a patient-centred focus with specific attention to the patient's bio-psychosocial needs, because of the vulnerability for misunderstandings of ethnic minority patients $[13,21]$. Our study showed that the doctors did not properly apply a number of specific ICC skills, such as adapting diagnosis and treatment policy to the cultural context. However, they also did not practice certain generic communication skills, such as exploring the reason for the consultation or checking the feelings of the patient, which is striking because we included medical specialists who could be expected to have learned how to practice these communication skills in their undergraduate and postgraduate training This is a valuable finding, as medical specialists function as role models for postgraduate trainees [22], which is, however, not always true due to the transfer problems of communication skills from training to trainee [23]. In all medical specialty training programmes in the Netherlands there is some formal communication training. However, this usually takes place during practice and not as additional training. The communication training is most extensive for residents of the training to become a general practitioner [23].

Nowadays, doctors in Western countries are taught to use a patient-centred communication style [8, 13, 21, 24]. Patient-centred communication has similarities with ICC, such as the responsibility of the doctor for the non-medical or interpersonal aspects of the communication [25]. The interpersonal aspects of care, for example trust, respect and empathy, are key determinants of patient satisfaction [13, 21]. As was mentioned above, we found missing generic communication skills, such as exploring the reason for the consultation, checking if the patient understood, and expectation management. These are skills of patient-centred communication as well [21]. In an intercultural context, patient-centred communication is probably even more important, because the balance in the interpersonal aspects of the communication is harder to find when doctor and patient have different norms and values. Intercultural and patientcentred communication have not been formally integrated together in medical education, although the function of both intercultural and patient-centred communication is to improve healthcare quality in similar ways and the used skills for patient-centred communication and ICC show similarities. Therefore, patient-centred communication and ICC should be incorporated in medical education, so that doctors will not have to learn two different approaches [13].

It was striking that the doctors all said they were satisfied with their conversation, while the observers concluded that doctors did not practice the relevant ICC skills. An explanation could be that the doctors need to be confronted with their communication behaviour before they can improve their communication skills [15]. Finally, we need to say that the complexity of ICC cannot be grasped in a list of do's and don'ts. It is not a matter of learning only one skill for ICC but of learning a complete set of skills and being able to apply these in the right way at the right time. It is the complete set of behaviours which makes a doctor a good intercultural communicator, and communication training is not a 'one size fits all' training [5]. 


\section{Conclusion}

We showed that doctors did practice some but not all the relevant ICC skills and that the ICC style of the doctors was mainly biomedically centred. Hence, it is unlikely that postgraduate medical trainees will acquire all the required ICC skills merely by modelling their behaviour on the example of their clinical supervisors. Furthermore, we discussed the possible overlap between intercultural and patient-centred communication. This overlap and the absence of skills in both these domains suggest that integrating patient-centred communication and ICC training may contribute substantially to the development of medical education for postgraduates and medical specialists.

\section{Strengths and limitations}

This observational study provided the opportunity to examine the application of ICC skills in real practice. A strength of this study was the focus on specialists instead of trainees, because medical specialists function as role models for postgraduate trainees. Another strength was that the consultations were videotaped before they were analyzed, and that the tapes were analyzed by observers from different areas of expertise, so that the data could be viewed from several perspectives. Since the groups of medical specialists were small, it was not possible to further analyze the influence of age and years of experience. Besides, the study population was too small to assess differences in communication styles between the doctors.

Another limitation was that we did not ask the patients for their years of residence in the Netherlands. The exact influence of this on ICC is hard to determine. On one hand, being in the Netherlands for a longer period could positively suggest an influence of the ICC with a native doctor. On the contrary, many people continue to identify themselves with their country of origin [26], which could suggest that years of residence in the Netherlands is less important.

\section{Implications for medical education}

Based on the results of our observational study of daily outpatient care and the points mentioned in the discussion, we would advise to extend the already existing communication training for postgraduate medical education with ICC-specific skills, such as asking about the language proficiency of patients or checking if the proposed treatment plan fits into the cultural habits of the patient. Elaborating ICC training could include discussion of doctors' own video consultations with peers in the presence of a communication expert. Besides, we would advise that medical specialists also embrace the concept of lifelong learning and that they attend communication training focused on patientcentred communication that includes ICC.

\section{Future research}

Many of the elements of the MAAS-Global ICC that we used seemed to be relevant for communication with every patient. Future research could study whether this is true, and should further explore the overlap between ICC and patient-centred communication. In the present study, we focused on the doctor from the third person, the observer. Also, it appears to be important to evaluate doctors' needs for ICC skills and patients' preferences and satisfaction with their doctors' communication skills. Further research could be a reflective practice study with doctors based on their videotaped consultations. Another further research possibility is to compare the patient-centred communication skills and ICC skills of doctors. This could facilitate the development of training focused on relevant communication skills.

Acknowledgements We are grateful to all the patients and doctors who participated in this study. Special thanks are conveyed to Lotte Roosendaal and Tugba Aydin, who helped with observing the data. We wish to thank Lisette van Hulst for editing the manuscript.

\section{Funding/Support None}

Conflict of interest E. Paternotte, F. Scheele, M.C. Seeleman, L. Bank, A.J.J.A. Scherpbier and S. van Dulmen declare that they have no competing interest, no financial competing interests and no nonfinancial competing interests.

The study was performed according to Dutch privacy legislation. Approval of the Dutch medical-education ethics board was obtained for this observational study (NVMO-ERB 355). Beforehand, all participating doctors and patients were informed about the aim and the procedure of the study. All participants signed an informed consent form before the recording of the consultation was started.

Open Access This article is distributed under the terms of the Creative Commons Attribution 4.0 International License (http:// creativecommons.org/licenses/by/4.0/), which permits unrestricted use, distribution, and reproduction in any medium, provided you give appropriate credit to the original author(s) and the source, provide a link to the Creative Commons license, and indicate if changes were made. 


\section{Appendix}

Table 4 MAAS-Global ICC observation scale

The doctor ...
Opening
Checks the language ability of the patient
Checks who is the formal speaker of the family
Asks to the relatives for their connection with the patient
Listens
Reacts adequately to possible cultural differences
Reason For Encouter

Demonstrates being alert to possible cultural aspects when asking for the reason for the consultation

Checks reasons of encounter of the relatives

Checks expectations regarding the consultation/healthcare

Physical Examination

Treats the patient with care and respect

Diagnosis

Explains cause and relation of the complaint within the context of the expectations of the patient

Checks if the patient and/or relatives understood the explanation

Policy

Adapt cultural differences in diagnosis and policy

Checks with the relatives if they understand the choice of policy

Makes appointments: who, what, when

Explains referral to other healthcare workers

Explore

Explores the reason for consultation, wishes and expectations

Explores the perception of the relatives

Recognizes misunderstanding caused by a language barrier

Explores the reaction of information transfer to the patient's context

Responds to non-verbal behaviour and keywords

Responds to cues/key words which are related to cultural differences

Emotions

Asks about the patient's feelings

Reflects on the feelings of the patient

Asks about the relatives' emotions

Listens actively

Tries to empathize the patient's emotions

Information Transfer

Checks the foreknowledge of the patient about diagnosis or expected policy

Gives information in small amounts

Gives concrete explanations

Uses concrete language

Asks if the patient understood the information

Uses different ways to give explanations

Pays attention to pronunciation

Uses attributes for explanation

Summarize

Summarizes the patient's story

Summarizes in his own words, concise

Attempts
Table 4 MAAS-Global ICC observation scale (Continued)

The doctor ...

Structure

Applies an adequate time schedule

Takes the time

Announces stages of the conversation

Empathy

Shows concern, is inviting and sincere, commiserates by means of eye contact and non verbal behavior, shows compassion for the patient

Commiserates with verbal reactions

Observes cultural differences

Shows empathic behavior

Has an open attitude

Shows respect for the patient

Consult Evaluation

Has an unprejudiced attitude

Demonstrates reliability

Shows awareness of his or her own cultural and professional context

Shows awareness of cultural differences

Speaks more languages of words of another language

Shows to have learned from previous consultations with ethnic minority patients

\section{References}

1. Teutsch C. Patient-doctor communication. Med Clin North Am. 2003;87:1115-45.

2. Schirmer JM, Mauksch L, Lang F, et al. Assessing communication competence: A review of current tools. Fam Med. 2005;37:184-92.

3. Betancourt JR, Green AR, Carrillo JE, Park ER. Cultural competence and health care disparities: Key perspectives and trends. Health Aff (Millwood). 2005;24:499-505.

4. Ferguson WJ, Candib LM. Culture, language, and the doctor-patient relationship. Fam Med. 2002;34:353-61.

5. Napier AD, Ancarno C, Butler B, et al. Culture and health. Lancet. 2014;384:1607-39.

6. Paternotte E, van Dulmen S, van der Lee N, Scherpbier AJJA, Scheele F. Factors influencing intercultural doctor-patient communication: A realist review. Patient Educ Couns. 2014; doi:10.1016/ j.pec.2014.11.018.

7. Schouten BC, Meeuwesen L. Cultural differences in medical communication: a review of the literature. Patient Educ Couns. 2006;64:21-34.

8. Teal CR, Street RL. Critical elements of culturally competent communication in the medical encounter: A review and model. Soc Sci Med. 2009;68:533-43.

9. Essers G, van Dulmen S, van Weel C, van der Vleuten C, Kramer A. Identifying context factors explaining physician's low performance in communication assessment: An explorative study in general practice. BMC Fam Pract. 2011;12:138.

10. Essers G, Dielissen P, van Weel C, van der Vleuten C, van Dulmen $\mathrm{S}$, Kramer A. How do trained raters take context factors into account when assessing GP trainee communication performance? An exploratory, qualitative study. Adv Health Sci Educ Theory Pract. 2015;20:131-47.

11. Essers G, van Dulmen S, van Es J, van Weel C, van der Vleuten C, Kramer A. Context factors in consultations of general practitioner 
trainees and their impact on communication assessment in the authentic setting. Patient Educ Couns. 2013;93:567-72.

12. Roberts C, Moss B, Wass V, Sarangi S, Jones R. Misunderstandings: A qualitative study of primary care consultations in multilingual settings, and educational implications. Med Educ. 2005;39:465-75.

13. Saha S, Beach MC, Cooper LA. Patient centeredness, cultural competence and healthcare quality. J Natl Med Assoc. 2008;100:127585.

14. Saha S, Beach MC. The impact of patient-centered communication on patients' decision making and evaluations of physicians: A randomized study using video vignettes. Patient Educ Couns. 2011;84:386-92.

15. van den Eertwegh V, van der Vleuten C, Stalmeijer R, van Dalen J, Scherpbier A, van Dulmen S. Exploring residents' communication learning process in the workplace: A five-phase model. PLoS ONE. 2015;10:e0125958.

16. Ram P, Grol R, Rethans JJ, Schouten B, van der Vleuten CP, Kester A. Assessment of general practitioners by video observation of communicative and medical performance in daily practice: issues of validity, reliability and feasibility. Med Educ. 1999;33:447-54.

17. Ram P, van Thiel J, van Dalen J. MAAS-Global Manual 2000. Maastricht: Maastricht University; 2003.

18. Streiner DL, Norman GR. Health measurements scales: A practical guide to their development and use, 4th ed. New York: Oxford University Press; 2008.

19. Veldhuijzen W, Ram P, van der Weijden T, Wassink M, van der Vleuten C. Much variety and little evidence: a description of guidelines for doctor-patient communication. Med Educ. 2007;41:138-45.

20. Hobma S, Ram P, Muijtjens A, van der Vleuten C, Grol R. Effective improvement of doctor-patient communication: a randomised controlled trial. Br J Gen Pract. 2006;56:580-6.

21. Mead N, Bower P. Patient-centredness: A conceptual framework and review of the empirical literature. Soc Sci Med. 2000;51:1087-110.
22. Watling C, Driessen E, van der Vleuten CP, Lingard L. The Accreditations Council for Graduate Medical Education (ACGME). Med Educ. 2012;46:192-200.

23. van den Eertwegh V, van Dulmen S, van Dalen J, Scherpbier AJ, van der Vleuten CP. Learning in context: Identifying gaps in research on the transfer of medical communication skills to the clinical workplace. Patient Educ Couns. 2013;90:184-92.

24. Ball JW, Dains JE, Flynn JA, Solomon BS, Stewart RW. Cultural competency. In: Mosby, editor. Seidel's guide to physical examination, 8th edn. Amsterdam: Elsevier; 2014. pp. 21-9.

25. Grol R, de Maeseneer J, Whitfield M, Mokkink H. Diseasecentred versus patient-centred attitudes: comparison of general practitioners in belgium, britain and the netherlands. Fam Pract. 1990;7:100-3.

26. Hofstede G, Hofstede GJ. Allemaal anders denkenden. Omgaan met cultuurverschillen, Business Contact ed. Amsterdam: Business Contact; 2005.

Emma Paternotte is a MD and a researcher in medical communication

Fedde Scheele is a gynaecologist and a professor in health systems innovations and medical education

Conny M. Seeleman is a researcher in the field of cultural competence

Lindsay Bank is a MD and a researcher in medical education

Albert J.J.A. Scherpbier is a MD and a professor in quality improvement in medical education

Sandra van Dulmen is a psychologist and a professor communication in healthcare 